\title{
A Numerical Analysis of Cold Powder Compaction Based on Micromechanical Experiments
}

\author{
E. Olsson ${ }^{\text {a }}$, P.-L. Larsson ${ }^{\mathrm{a}, *}$ \\ ${ }^{a}$ KTH Royal Institute of Technology, Department of Solid Mechanics, Osquars Backe 1, 10044 Stockholm, Sweden
}

\begin{abstract}
The Discrete Element Method (DEM) is used for predicting the compaction behavior of two types of spray dried cemented carbide granules. The material model of the granules is determined by micromechanical experiments. Firstly, compression tests are made on single granules giving information of the deformation behavior at relatively small deformations. For larger deformations, nanoindentation tests are made to give further information of the constitutive behavior which shows a strong hardening behavior at high strains. The material model is implemented in a FE model of two particles in contact and the relation between contact force and indentation depth is exported to a DEM program. The DEM program is used to simulate presently performed uniaxial die compaction experiments where the geometry of the die is taken into account. Excellent agreement is found between the experiments and the numerical predictions in the range where results from DEM simulations are valid.
\end{abstract}

Keywords: Powder Compaction, Granule Strength Measurements, Contact Mechanics, Discrete Element Method, Cemented Carbides, Nanoindentation

\section{Introduction}

Die powder compaction followed by sintering is a common process route in the production of hard metal components and components with complex shape. The compaction process can be divided into three stages. The first stage, sometimes denoted stage 0 , is the filling process where the powder is filled into the pressing die. In the second stage, stage I, the powder is compacted with plastic deformation of the powder particles and this is the main focus of the present work. In this stage, the contact areas between the particles are small and can thus be treated independently. This stage ends at packing densities around $80 \%$ to $90 \%$. In the last stage, stage II, the compact is pressed up to (almost) full density and the compact behaves mechanically like a porous solid.

\footnotetext{
* Corresponding author

Email addresses: erolsson@kth.se (E. Olsson), pelle@hallf.kth.se (P.-L. Larsson )
} 
When modeling stage I compaction, a micromechanical model is beneficial as then the analysis is based on a careful description of the mechanical behavior of the powder particles instead of relying on the more phenomenological models concerning porous solids. In the field of micromechanical modeling, pioneering work was presented by Wilkinson and Ashby [1] followed by investigations by Fleck et. al [2] and Fleck [3]. It is essential in a micromechanical model that the contact between two powder particles are modeled in an adequate manner. One approximation that makes it possible to obtain an analytical solution is that the particles are assumed to be spherical which is the case for atomized powder particles or spray dried granules. This problem of two spheres in contact was studied analytically using similarity methods by Biwa and Storåkers [4] and later by Storåkers et. al [5] and Storåkers [6] taken into account plastic flow, viscoplasticity and creep. These models was later utilized in analytical studies of powder compaction by Larsson et. al [7] and Storåkers et. al [8]. The effect of adhesion between two ideal-plastic particles was included by Mesarovic and Johnson [9]. Recent studies of the problem with two particles in contact includes more general studies by Skrinjar et. al [10] and Olsson and Larsson [11] where elastic-plastic materials, strain hardening and adhesive effects are investigated. A sketch of a typical normal force contact relation is shown in Figure 1 and the major issue in this work is to determine this relation based on experimental results.

The analytical studies of powder compaction mentioned above were all based on some simplifying assump-

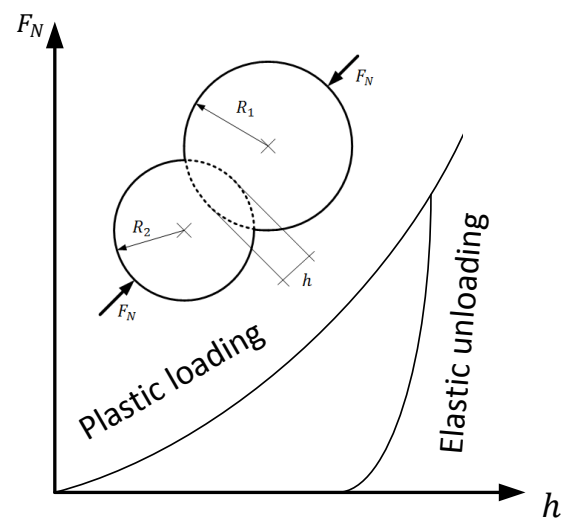

Figure 1: Sketch of the behavior of the normal contact force at loading and unloading

tions and in particular then the assumption of affine motion. This assumption can be avoided in compaction studies by using the Discrete Element Method (DEM). Work in this field started by Heylinger and McMeeking [12], followed by Martin et. al [13], Martin and Bouvard [14] and Skrinjar and Larsson [15, 16].

In all these studies, both analytical and DEM simulations, the mechanical properties of the powder particles are assumed to be known and is utilized in evaluating the contact relations from the investigations mentioned above. However, for many industrially relevant applications, the mechanical properties are not known on the granular scale. This includes for instance spray dried powder granules where very fine particles, $<1 \mu \mathrm{m}$, 
are mixed with a binder and spray dried to larger granules $\approx 10-100 \mu \mathrm{m}$ in order to enhance handability. In order to overcome this problem, Pizette et. al [17] performed an interesting study where they assumed a specific form of the force-displacement relation for two contacting particles. The unknown parameters were then identified by comparing numerical data and experimental compaction results using a Design of Experiment (DoE) setup.

In this work, a more direct method is used to determine the contact relation between two powder particles. Two different kinds of experiments are performed on single particles, a compression test and a nanoindentation test. The material parameters evaluated from these tests are then implemented in a FE model with two contacting granules and the resulting force-displacement relation is exported to the DEM program. DEM simulations is then done and will be compared with results from compaction experiments.

The experimental part of the compaction analysis is limited to two different spray dried cemented carbide powders used in the industry, with slightly different compaction behavior and composition. Here and in the sequel the Powders are identified as Powder A and Powder B. The granules is manufactured by mixing small tungsten carbide and cobalt particles with a polymeric binder which is then spray dried resulting in a complex material behavior. The aim, in short then, is to predict the (uniaxial) compaction behavior using data from micro mechanical experiments together with DEM simulations. To the authors knowledge, no previous experimental studies have been performed with the intention to explicitly study normal contact force models for powder granules.

\section{Micromechanical Experiments}

To simulate the compaction of the cemented carbide granules, the normal contact force $F_{N}$ as function of the indentation depth $h$ needs to be known. In order to achieve this, two different types of experiments are performed on single granules. These experiments are used to determine the details of the constitutive behavior of the material of the granules. This model is then implemented in a finite element model of two granules in contact from which the $F_{N}(h)$ relation can be extracted.

The mechanical behavior of the powder granules is assumed to be governed by classical elastoplasticity described by Young's modulus $E$, Possion's ratio $\nu$ and von Mises' type plasticity with isotropic hardening. Hence, possible porosity of the granules is neglected in the material model but could be taken into account using a more elaborate description for instance the Drucker-Prager or the Gurson-Tvergaard models.

\subsection{Granule compression experiments}

A granule strength measurement system manufactured by etewe [18] is used in the first type of experiments performed on cemented carbide granules. The granule is compressed between two plates (which are assumed to be rigid compared to the granules). One benefit of this test is that it, at least initially, is similar to the contact situation between two granules during powder compaction. 
Prior to the testing, the diameters of the granules in the $x$ and $y$ directions, defined in Figure 2, $D_{x}$ and $D_{y}$, are measured automatically using an image identification system. The diameter in the $z$ direction, $D_{z}$, is defined as the distance from the bottom plate when the upper plate starts to take load. The experiments are performed in a controlled load setting and is continued until fracture of the particle. During the test, the compression force $F$ and and movement of the upper plate, $2 \Delta$, are registered giving a deformation of $\Delta$ at each contact point.

The tests were performed on 80 granules of powder $\mathrm{A}$ and 60 of powder $\mathrm{B}$ and were made on particles of

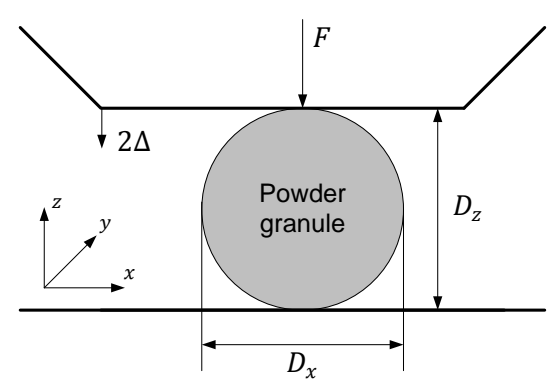

Figure 2: Sketch of the granule compression test setup

different sizes. It was thought advisable to use the most spherical granules in the determination of mechanical properties and hence a sphericity criterion was defined as

$$
\frac{\sqrt{\left(D_{x}-D\right)^{2}+\left(D_{y}-D\right)^{2}+\left(D_{z}-D\right)^{2}}}{D} \leq 2.5 \%
$$

where $\mathrm{D}$ is the mean diameter $D=\left(D_{x}+D_{y}+D_{z}\right) / 3$ and thus the mean radius is $R=D / 2$. After this filtering, 9 granules from Powder A and 12 granules of Powder B were included in the analysis below.

The outcome of the granule compression experiments is presented in Figure 3. Initially, the results for Powder $\mathrm{A}$ and Powder $\mathrm{B}$ coincide but at a deformation $\Delta / R=0.02$, the granules in Powder $\mathrm{B}$ are behaving softer than granules from Powder A. This could be explained by initiation of micro cracks or less homogeneous granules in Powder B. The force-displacement relation for both sets of powders is initially close-to linear which according to the self-similarity solution $[5,6]$ indicates a ideal-plastic material with negligible elastic deformation. The force-displacement relation becomes under such circumstances

$$
\frac{F}{R^{2}}=\alpha \pi c^{2} \sigma_{Y} \frac{\Delta}{R}
$$

where, according to previous work [11], $\alpha=5.6$ for a sphere against a rigid plane and $\alpha=6$ for a rigid sphere indenting a deformable material (Brinell hardness test). The area parameter $c^{2}$ takes on the value $c^{2}=1.43$ for ideal-plastic materials with negligible elastic deformation [4]. This linear fit is shown by dashed lines in Figure 3 and gives a value on the yield stress of $\sigma_{Y}=5.8 \mathrm{MPa}$ for both Powder $\mathrm{A}$ and Powder B. However, the self-similarity model predicts a too stiff response after the most initial part of the tests compared with 

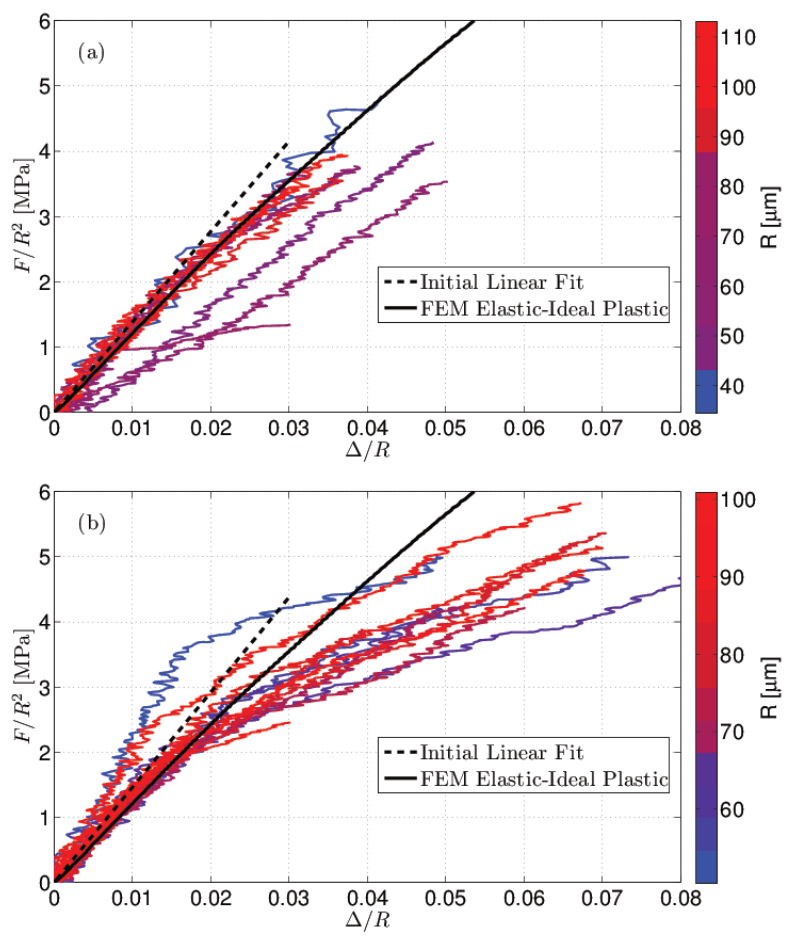

Figure 3: Compression force as function of displacement from the granule compression experiments for (a) Powder A, and (b) Powder B. A linear fit to the initial part of the curves and results from the finite element simulations are also included

the experimental data.

In order to bring more light into this discrepancy, the contact problem was analyzed with the Finite Element Method (FEM) using the multi-purpose FE software ABAQUS [19]. The meshed model is shown in Figure 4 and consists of 51218 nodes and 25906 axi-symmetric elements. The mesh was made denser and regular in the contact region with a smallest element length of $1 / 400$ of the radius of the particle for increased accuracy. The elements were of hybrid type in order to improve convergence at high plastic strains. The FE simulations were done using frictionless contact knowing that friction has a negligible effect on global indentation properties [20]. An elastic-ideal plastic material model was used with $\sigma_{Y}=5.8 \mathrm{MPa}$ and to have negligible elastic deformation, a value of $E=1000 \sigma_{Y}$ was used for the Young's modulus.

The result of the FEM simulations is shown as solid lines in Figure 3. As seen in Figure 3 (a) the simulation and the results from the experiments agrees very well for Powder A whereas these is some discrepancy for Powder B. This can, as mentioned earlier, perhaps be explained by less homogeneous granules or micro cracks in the case of Powder B. The difference between the simulations and the self-similarity solution, Eq. 2, is due to large deformation effects which were studied in detail by Mesarovic and Fleck [21, 22].

The FE model was run several times with a strong linear hardening appearing at a specific value of the plastic strain. The results were compared with the ideal plastic model with the aim to get the same results for deformations below $\Delta / R=0.04$ which is the range relevant for the experiments. The conclusion of this 
(a)

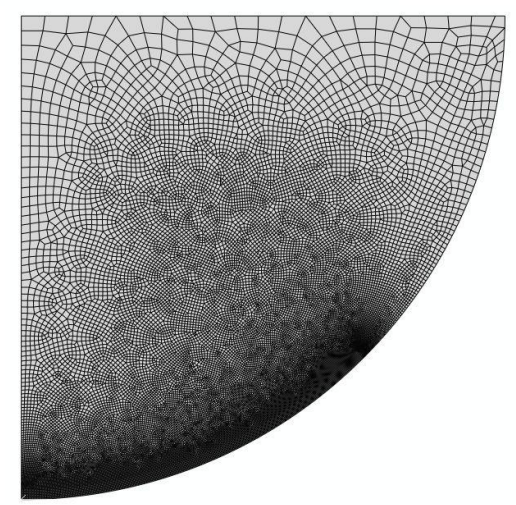

(b)

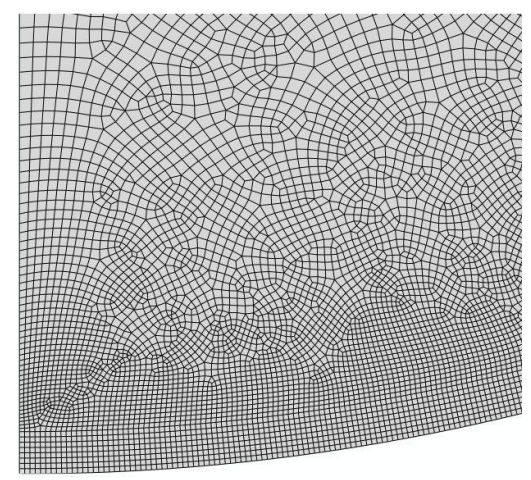

Figure 4: Finite element mesh used in the analysis of the granule compression test (a) Complete mesh. (b) Magnified part of the contact region

investigation is that the material of the granules could be approximated to be elastic-ideal plastic up to a plastic strain of $17 \%$. Above this level, the granule compression experiments give no further information of the material behavior.

\subsection{Nanoindentation experiemnts}

Previous work devoted to the same materials as used here, Andersson et. al [23] and Lindskog et. al [24] shows a strong deformation hardening effect during compaction. Thus it is expected that an elasticideal plastic material model is not sufficient to describe the material of the cemented carbide granules and according to the discussion in the previous section, more data is needed for plastic strains above $\varepsilon_{p l}=17 \%$. One type of testing providing information at such high strains without deforming a too large volume and thus breaking the granules, is nanoindentation testing. In a nanoindentation test, a tetrahedral tip is pressed into the material and the indentation force, $P$, is registered as function of the indentation depth $h$. Such test is often called Berkovic indentation and this contact problem was analyzed in detail by Larsson et. al $[25]$.

In order to be able to handle the powder granules in the testing equipment, the granules were embedded in epoxy and the surface of the granule-epoxy cylinder was then polished to get well defined inprints. The 
polished surface with two indentation marks in Powder B is shown in Figure 5.

The measurements were performed using load control. Firstly, the load increased monotonically up to an

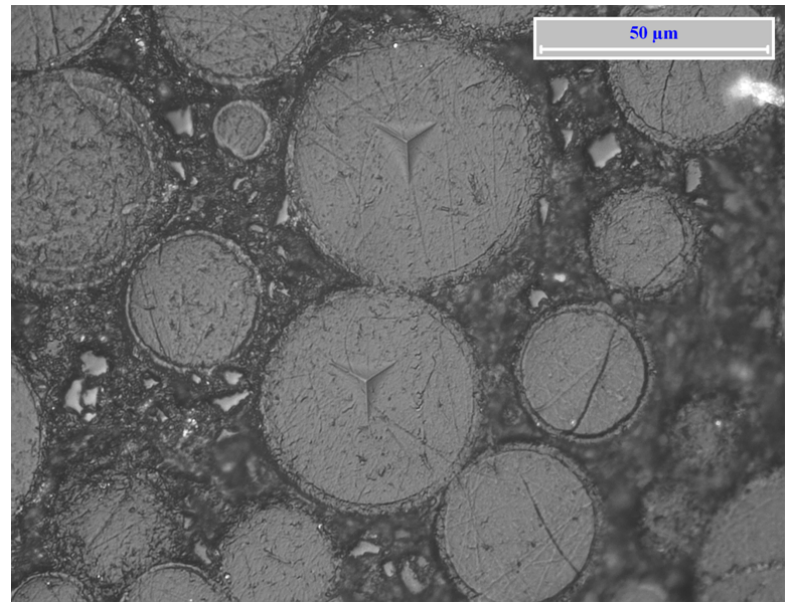

Figure 5: Micrograph of the marks of two nanoindentation tests performed on granules of Powder B

indentation depth of $2 \mu \mathrm{m}$ and were then kept constant for $20 \mathrm{~s}$. An indentation depth of $2 \mu \mathrm{m}$ was chosen as a compromise between having a sufficiently large indent to get an average over the microstructure of the granules and a sufficiently small indent to be able to use formulas for infinite half spaces in the evaluation of the experiments. After that the tip is unloaded to the point where $1 / 5$ of the maximum force remains. There the force is held constant in $2 \mathrm{~s}$ before continued unloading until the tip leaves the surface. The resulting force-displacement curves for this sequence is shown for all performed tests in Figure 6.

The plateaus at constant load in the curves shown in Figure 6 shows a creeping behavior. This feature is of course very relevant for a high accuracy analysis but is presently deemed to be a secondary effect and is not considered further. The indentation hardness is calculated from

$$
H=\frac{P}{A_{p}}
$$

where $P$ is the maximum force and $A_{p}$ is the projected contact area calculated automatically in the experimental equipment by carefully calibrating the equipment against a material with known hardness. The formula for the contact area can be found in Oliver and Pharr [26]. One issue in determining the hardness from an instrumented nanoindentation test is that the contact area calculated from the measured data and the optically measured area might differ as discussed in Tsui et. al [27]. In order to account for this possibility, the contact area was estimated from the indentation marks in Figure 5 and the difference was found to be very small.

The average hardness together with the standard deviation is presented in Table 1. Powder A has a somewhat higher hardness then Powder B and the scatter in the data for Powder B is much higher than for Powder A. This is expected based on the results from the granule compression tests where the granules from 

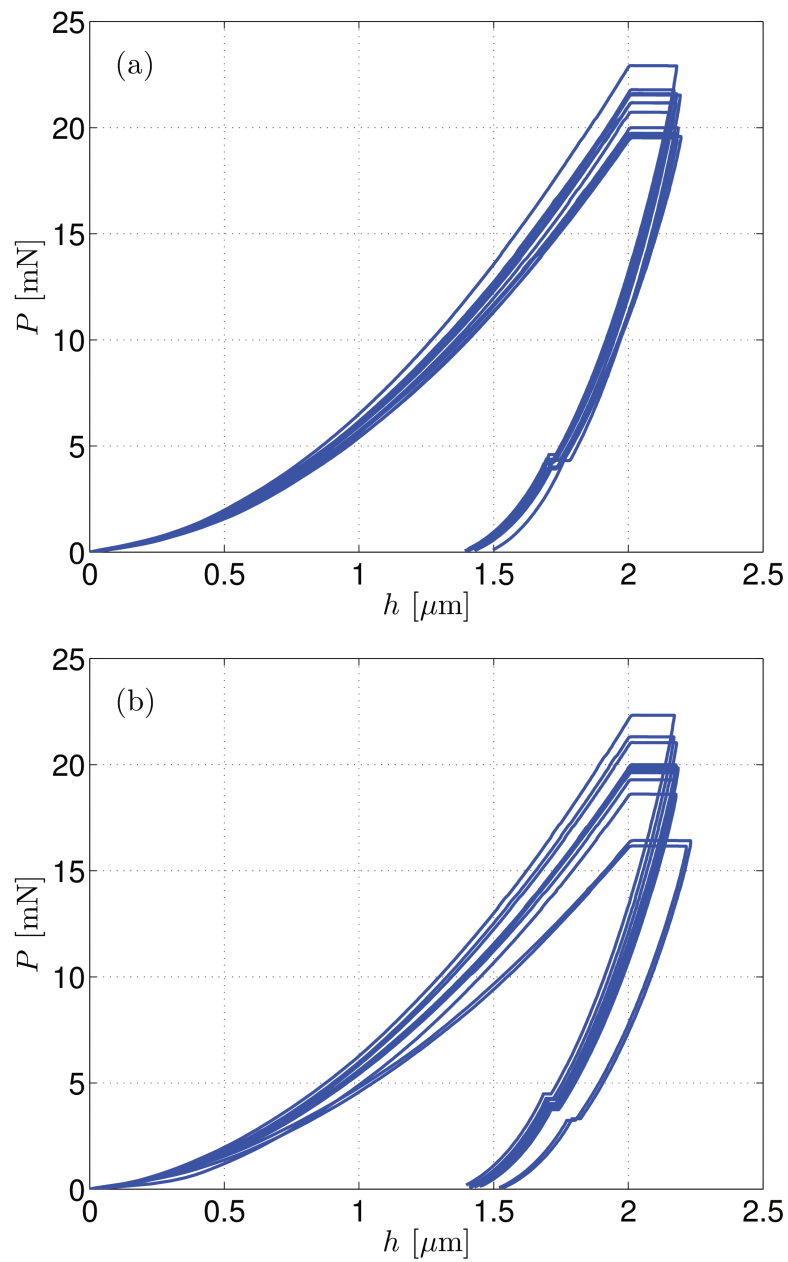

Figure 6: The indentation force as function of the indentation depth during the nanoindentation tests of the powder granules. Data for Powder A are presented in (a) and data for Powder B in (b)

Powder B was found to be less homogeneous.

Young's modulus of the indented material can be determined by use of the initial unloading section of the measured force-displacement using the relation [26].

$$
E^{*}=\frac{1}{C} \frac{\sqrt{\pi}}{2} \frac{1}{\sqrt{A_{p}}} \frac{d P}{d h}
$$

where $C$ is a constant that takes on the value 1.034 for a Berkovic indenter and $E^{*}$ is the effective elastic modulus defined as

$$
E^{*}=\frac{E}{1-\nu^{2}}
$$

where the compliance of the indenter has been neglected. In order to get the elastic modulus $E$ for the indented material one needs to guess the Possions' ratio $\nu$ which was set to 0.3 in the following. However, the quotient $E /\left(1-\nu^{2}\right)$ appears instead of $E$ alone for elastic and elastic-plastic [11] contact relations and 
Table 1: Material data evaluated from the nanoindentation tests

\begin{tabular}{lccc}
\hline Powder & $H[\mathrm{MPa}]$ & $E[\mathrm{GPa}]$ & $\sigma_{0.35}[\mathrm{MPa}]$ \\
\hline Powder A & $234 \pm 12$ & $5.72 \pm 0.53$ & 161 \\
Powder B & $215 \pm 25$ & $5.90 \pm 0.69$ & 148 \\
\hline
\end{tabular}

thus the results in this paper should not be much dependent on the assumed value of $\nu$. The calculated values of $E$ are presented in Table 1. The scatter in the determination of Young's modulus is much higher then the scatter for the hardness, probably due to difficulties in determining the unloading stiffness $d P / d h$. Also here, the values for Powder B has a bigger standard deviation than correspondingly for Powder A. Finally, the calculated values of Young's modulus agrees well with the assumption of negligible elastic deformation during the granule compression tests with $E / \sigma_{Y} \approx 1000$.

The hardness can be related to the flow stress at some representative value of the plastic strain. For a material with a regular strain hardening, the Berkovic hardness is approximately three times the yield stress at $8 \%$ plastic strain. However, for a material with an irregular stress-strain curve, as expected here, Larsson [28] discovered that a single stress value is not sufficient to describe the hardness. Furthermore, the following expression was found from extensive FE simulations to give a good prediction.

$$
H=C_{1} \sigma_{0.02}+C_{2} \sigma_{0.35}
$$

where $\sigma_{0.02}$ is the flow stress at a low value of the plastic strain, $\varepsilon_{p l}=2 \%$, and $\sigma_{0.35}$ is the flow stress at a high value of plastic strain, $\varepsilon_{p l}=35 \%$. Furthermore, the parameters $C_{1}$ and $C_{2}$ where determined to be $C_{1}=C_{2}=1.4$ for a Berkovic indenter. The computed value of the flow stress at $35 \%$ plastic strain, $\sigma_{0.35}$, are included in Table 1 based on the previous result that $\sigma_{0.02}=\sigma_{Y}$. In summary then, the granules are assumed to be constitutively described by an elastic plastic material which behaves ideally plastic up to a plastic strain of $\varepsilon_{p l} \geq 17 \%$ but have a rapid strain hardening in the range $\varepsilon_{p l} \geq 17 \%-35 \%$. Hence, the following constitutive model is assumed

$$
\sigma= \begin{cases}\varepsilon E & \varepsilon E \leq \sigma_{Y} \\ \sigma_{Y} & \sigma_{Y} / E \leq \varepsilon_{p l} \leq \varepsilon_{H} \\ \sigma_{Y}+\sigma_{0}\left(\varepsilon_{p l}-\varepsilon_{H}\right)^{1 / m} & \varepsilon_{p l} \geq \varepsilon_{H}\end{cases}
$$

In order to fulfill the measured value of $\sigma_{0.35}, \sigma_{0}$ is given by

$$
\sigma_{0}=\frac{\sigma_{0.35}-\sigma_{Y}}{\left(0.35-\varepsilon_{H}\right)^{1 / m}}
$$

The model contains two unknown parameters, the plastic strain at the onset of hardening, $\varepsilon_{H}$, which lies in the range $0.17-0.35$, and the plastic hardening exponent $m$. 
Table 2: Properties of the two investigated powders

\begin{tabular}{lccccc}
\hline Powder & $D_{t h}\left[\mathrm{~g} / \mathrm{cm}^{2}\right]$ & $D_{\text {bulk }}\left[\mathrm{g} / \mathrm{cm}^{2}\right]$ & $D_{\text {tap }}\left[\mathrm{g} / \mathrm{cm}^{2}\right]$ & $\theta_{\text {resp }}\left[{ }^{\circ}\right]$ & $\mu_{\text {part }}$ \\
\hline Powder A & 14.45 & 3.554 & 3.8 & 30 & 0.58 \\
Powder B & 14.64 & 3.382 & 3.571 & 32 & 0.62 \\
\hline
\end{tabular}

\section{Compaction Experiments}

The compaction behavior of the two powders was investigated in a powder testing equipment from PTC [29]. A sketch of the test setup is shown in Figure 7. The density of a final sintered component, called $D_{t h}$, will be used as the reference density and is reported in Table 2.

Before the compaction experiments, the density directly after filling and the density after shaking the
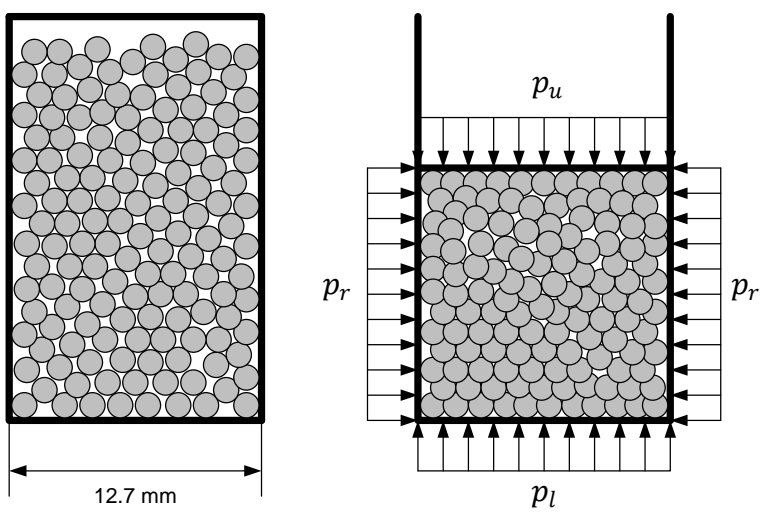

Figure 7: Sketch of the compaction test setup

powder container was measured and both are reported in Table 2. In order to get an estimate of the friction between the particles the angle of response $\theta_{\text {resp }}$ was also measured. The friction coefficient, $\mu_{\text {part }}$ can then be estimated as

$$
\mu_{\text {part }}=\tan \theta_{\text {resp }}
$$

relying on a standard Coulomb friction model. It can be noted that the friction coefficient is almost the same for both powders but the filling densities is notably lower for Powder B. Hence, it is concluded that the actual density of the granules is lower for Powder B than for Powder A.

The powder was pressed up to a relative density (mass density of compact divided by $D_{t h}$ ) of $60 \%$ even though the relevant range for discrete element simulations ends at a density about $45 \%$. The compaction was made in single action uniaxial pressing with the bottom punch fixed and a movable upper punch with a continuous measurement of the pressure on both punches. The final cylindrical compact had a diameter of $12.7 \mathrm{~mm}$ and a height of $10 \mathrm{~mm}$. Two compaction tests was made on each powder and no difference was found between them. The result of all four experiments is shown in Figure 8 in the range of the relative density 
relevant for DEM simulations. A slightly lower pressure is needed to obtain a specific density for Powder

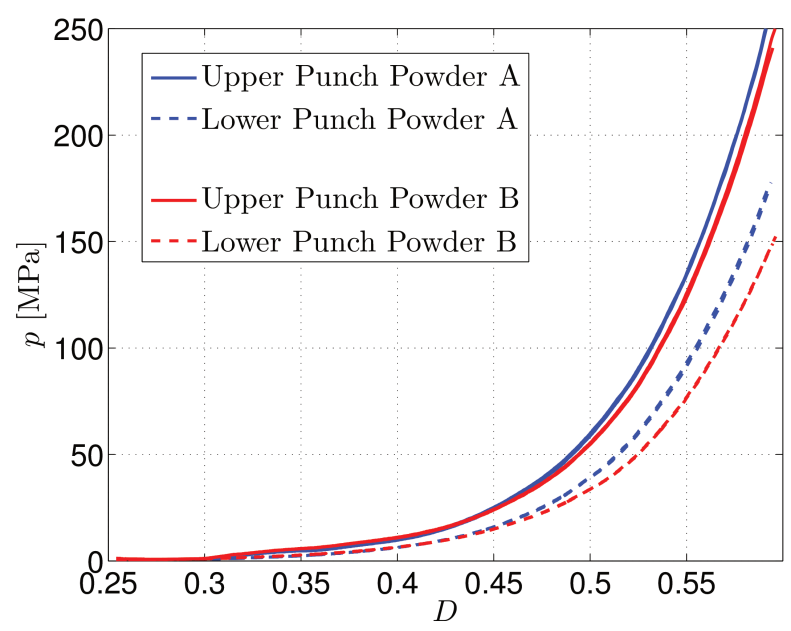

Figure 8: The experimentally determined relation between uniaxial compaction pressure and relative density for Powder A and Powder B

B then for Powder A which is in agreement with the slightly lower hardness value for Powder B. At low densities, $D \leq 0.4$, the compaction behavior is almost identical which corresponds well with the results from the granule compression tests. The relative density of a single granule is important when comparing results from the simulations and the experiments. This property was determined from initial DEM simulations by determining when the powder compact starts to take load, i. e when the assembly of particles behaves like a solid under compression and compare it with the experiments. This study suggested that the granules of Powder A have a relative density of $48 \%$ and granules of Powder B have a slightly lower density, $46 \%$.

\section{Simulations}

\subsection{Discrete Element Method}

In the Discrete Element Method (DEM), developed by Cundall and Strack [30], each particle is modeled as a separate object and the local contact forces acting between the particles determines the motion of the particles. Hence, determining the contact force is the most critical issue for the macroscopic behavior and will thus be discussed in detail in the next section.

The simulations start with randomly placing particles in the container at a low packing density of $10 \%$. Thereafter, compaction starts by moving the container walls with slow constant velocities. In this case, where single action uniaxial pressing is simulated, only the upper wall is moved. A more realistic way to simulate the filling process was tried in previous work, [31] but didn't notably change the macroscopic behavior more than at low relative densities, $30 \%-33 \%$.

To calculate the position, $\mathbf{x}_{i}$, and velocity, $\mathbf{v}_{i}$, of particle $i$ at a time $t+\Delta t$, Newton's second law was 
integrated explicitly using a Verlet type algorithm.

$$
\begin{aligned}
& \mathbf{x}_{i}(t+\Delta t)=\mathbf{x}_{i}(t)+\left(\mathbf{v}_{i}(t)+\frac{1}{2} \mathbf{a}_{i}(t) \Delta t\right) \Delta t \\
& \mathbf{v}_{i}(t+\Delta t)=\mathbf{v}_{i}(t)+\frac{\mathbf{a}_{i}(t)+\mathbf{a}_{i}(t+\Delta t)}{2} \Delta t \\
& \mathbf{a}_{i}(t+\Delta t)=\frac{\mathbf{F}_{i}(t)}{m_{i}}
\end{aligned}
$$

with $\mathbf{F}_{i}(t)$ being the sum of forces, normal and frictional, acting on particle $i$ and $m_{i}$ is the mass of particle $i$. The rotational motion of the particles is also considered and the rotation angle, $\boldsymbol{\theta}_{i}$ and angular velocity $\boldsymbol{\omega}_{i}$ of particle $i$ is calculated in a similar manner by

$$
\begin{aligned}
\boldsymbol{\theta}_{i}(t+\Delta t) & =\boldsymbol{\theta}_{i}(t)+\left(\boldsymbol{\omega}_{i}(t)+\frac{1}{2} \boldsymbol{\alpha}_{i}(t) \Delta t\right) \Delta t \\
\boldsymbol{\omega}_{i}(t+\Delta t) & =\boldsymbol{\omega}_{i}(t)+\frac{\boldsymbol{\alpha}_{i}(t)+\boldsymbol{\alpha}_{i}(t+\Delta t)}{2} \Delta t \\
\boldsymbol{\alpha}_{i}(t+\Delta t) & =\frac{\mathbf{M}_{i}(t)}{I_{i}}
\end{aligned}
$$

with $\mathbf{M}_{i}(t)$ being the sum of moments on particle $i$ from the frictional forces and $I_{i}=2 m_{i} R_{i}^{2} / 5$ is the moment of inertia of the particle.

In order to ensure numerical stability of the explicit integration method, it was shown by Cundall and Strack $[30]$ that a maximum time step $\Delta t$ of

$$
\Delta t<2 \sqrt{\frac{m_{\min }}{k}}
$$

is required where $m_{\min }$ is the mass of the smallest particle and $k=d F / d h$ is the contact stiffness. This stability criterion gives a far too small time step and in order to reduce the computational time to a reasonable level, it was shown by Thornton and Antony [32], that under quasi-static conditions, the masses could be upscaled several orders of magnitude without affecting the macroscopic response. In these simulations, a scale factor of $10^{11}$ was chosen. Also, in order to improve stability, a velocity limitation criterion was set on each particle requiring that

$$
\left\|\mathbf{v}_{i}\right\|=\min \left(v_{\max },\left\|\mathbf{v}_{i}\right\|\right)
$$

where $v_{\max }$ was chosen to be five times the speed of the die walls.

\subsection{Contact model}

The most critical issue in a DEM simulation is the description of the contact force between two particles. In a compaction simulation, the computation needs to be very accurate in order to get the correct compaction pressure but the computations also need to be very fast due to the fact that the contact relations are computed billions of times. In order to achieve this, a FE simulation was made prior to the DEM simulations 
using the same model as described for the granule compaction tests but continued up to a much higher overlap, $h / R=0.3$. The evaluated points on the $F-h$ curve, a few hundreds, were then loaded in the DEM program and a linear interpolation scheme was used to compute the normal force $F$ at a specific overlap $h$. One benefit with using data directly from FEM is that effects of large deformations are explicitly taken into account.

In order to determine the unknown parameters in the constitutive model, $m$ and $\varepsilon_{H}$, DEM simulations were performed with a low number of particles $(N=1000)$. Comparing the curve shapes from these simulations and the compaction experiments suggested that the hardening must be strong and thus the power law exponent should be high, $m \geq 20$, giving almost a step in the yield stress as function of the plastic strain at $\varepsilon_{H}$. Furthermore, by studying the onset of noticeable hardening at compaction, $\varepsilon_{H}$ was suggested to be 0.21 for Powder A and higher, 0.23, for Powder B which agrees with the assumption that the granules in Powder A are denser. It should be mentioned that some parameter fitting is required to get reliable results from the simulations, but the effort in this fitting is significantly reduced using the results from the micromechanical experiments. The stress as function of the total strain is presented in Figure 9 (a) for both powders and the resulting force-displacement relations are presented in Figure 9 (b) where the strong hardening is clearly visible. Unloading of the two spheres in contact is a more complicated issue due to the fact that unloading is dependent on the loading history. A fairly general analysis was presented in Olsson and Larsson [11] but any explicit formulas for direct implementation in a DEM program could not be derived and would require some complicated curve fitting. In this case, the main purpose of studying elastic unloading is to introduce damping in the model, and not to give any quantitative prediction of elastic spring back etc, and thus a more approximate model is sufficient. In the following, any adhesive effects are neglected in order to simplify the analysis but could be included using the results in [9] or [11].

If the contact pressure is constant, the analytical solution by Mesarovic and Johnson [9] could be used during unloading. This is a very good approximation for ideal plastic materials but not for strain hardening materials. Hence, the equations below should accurately describe the unloading during initial compaction but will fail during the later stages due to the strong hardening behavior. The model in [9] relates the recovered overlap, $h_{u}=h_{\max }-h$ to the contact radius $a$ and further, $a$ to the force during unloading $F_{u}$ by

$$
\begin{aligned}
h_{u} & =\frac{4 p_{0} a_{0}\left(1-\nu^{2}\right)}{E} \sqrt{1-\left(\frac{a}{a_{0}}\right)^{2}} \\
F_{u} & =\frac{2 F_{0}}{\pi}\left[\arcsin \left(\frac{a}{a_{0}}\right)-\left(\frac{a}{a_{0}}\right) \sqrt{1-\left(\frac{a}{a_{0}}\right)^{2}}\right]
\end{aligned}
$$

for spheres of equal material. The (constant) contact pressure takes on the value $2.8 \sigma_{Y}$, and $F_{0}$ and $a_{0}$ are contact force and contact radius prior to unloading. In the DEM simulations, $a_{0}$ is determined in the same way as the normal force during loading, by linear interpolation of data from the FE simulations. 

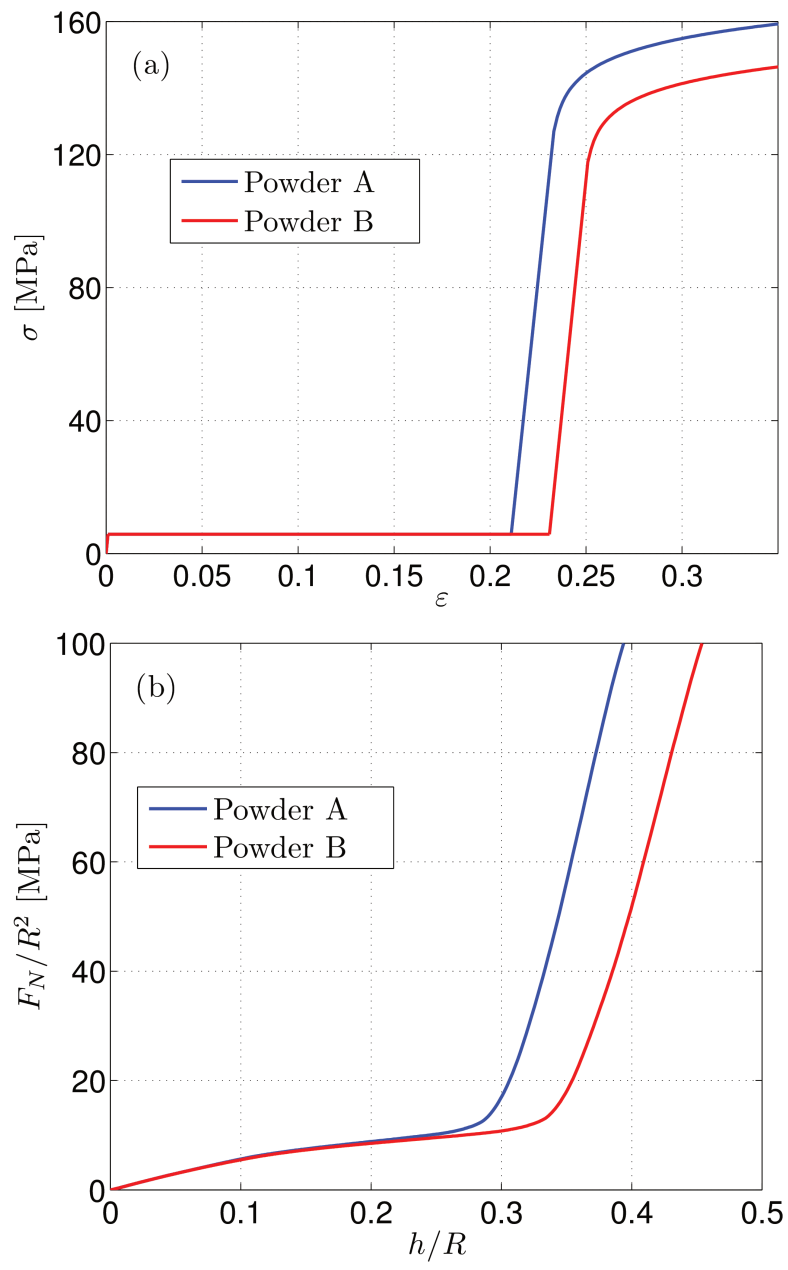

Figure 9: (a) Suggested material model for the powder granules. (b) The normal contact force as function of the indentation depth for contact between two granules.

Finally, the model for the frictional forces should be discussed. In this work, a stick-slip friction model is used both for contacts between two particles and between a particle and a wall. Using this model, the tangential force, $\boldsymbol{F}_{T}$ is proportional to the displacement of the center of the particle in the direction parallel to the contact plane, $\boldsymbol{\delta}_{t}$, for sticking contact or modeled by Coulomb friction in the sliding state. This model could be described mathematically by:

$$
\boldsymbol{F}_{T}= \begin{cases}-k_{T} \boldsymbol{\delta}_{t} & k_{T}\left\|\boldsymbol{\delta}_{t}\right\| \leq \mu F_{N} \\ -\mu F_{N} \frac{\boldsymbol{\delta}_{t}}{\left\|\boldsymbol{\delta}_{t}\right\|} & k_{T}\left\|\boldsymbol{\delta}_{t}\right\| \geq \mu F_{N}\end{cases}
$$

where $F_{N}$ is the normal contact force, $\mu$ the coefficient of friction and $k_{T}$ is the tangential stiffness. Despite the fact that the stick slip model is often used in the literature, explicit values of the tangential stiffness are rare. In this work a value of $k_{T}=10^{4} \mathrm{~N} / \mathrm{m}$ was used which is very close to the initial normal stiffness given by the similarity solution, Eq. 2. The friction coefficient was set to $\mu_{\text {part }}$ in Table 2 for contacts between two 
particles. Previous studies on pertinent materials suggested that the friction coefficient between the powder and the die walls could be rather low $\left(\mu_{\text {wall }} \approx 0.2\right.$ ), Samuelson and Bolin [33], but also varying from high values down to $\approx 0.2$ during compaction, Wikman et. al [34]. Hence, a small parameter study was made by investigating three different values on $\mu_{\text {wall }}, \mu_{\text {wall }}=0.2,0.3$ and 0.4 .

\subsection{Simulation results}

The uniaxial compaction experiments is simulated in DEM taking the actual geometry into account by having the same quotient between the die height and the die radius. By having sufficiently many particles in the simulation, it should then be possible to predict the pressure on both the upper and lower punch. This in contrast to using a representative volume element with periodic boundary conditions where only a mean pressure can be extracted.

The simulations were done with 8000 particles of equal size knowing that the effect of particle size distribution is small [31]. Some simulations were performed with half and also doubled amount of particles with no visible difference. The powder compact at the density where the pressure starts to increase and at a higher density are shown in Figure 10. The results from the simulations together with the data from the compaction
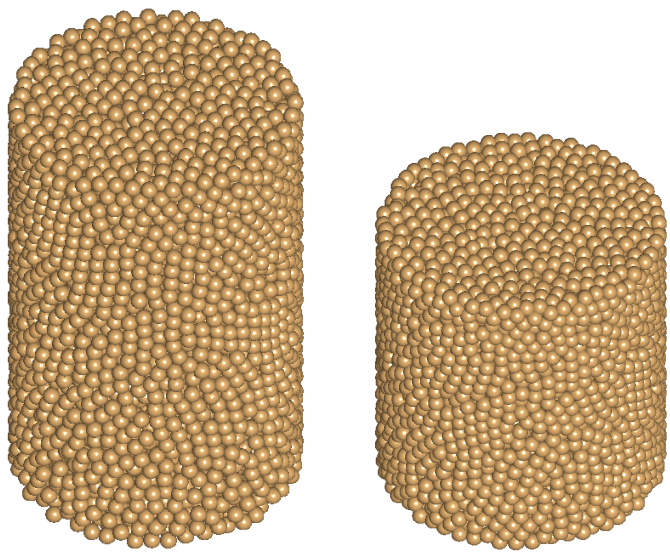

Figure 10: (a) A random assembly of 8000 particles at the density where the compact starts to take load, $\mathrm{D}=0.3$. (b) The compact pressed to a density of $\mathrm{D}=0.44$.

experiments are shown in Figure 11. The agreement between the simulations and the experiments is very good up to $D \leq 0.45$ where among other things the assumption of independent contacts is not valid anymore. The results clearly indicates that the wall friction coefficient lies in the range of $0.2-0.3$ with almost perfect agreement for $\mu_{\text {wall }}=0.2$ which is the value reported in [33]. One effect that could not be studied in the experiments is the pressure in the radial direction, $p_{r}$. It is therefore interesting to note that the simulations show that $p_{r}$ is almost independent of the wall friction and thus the pressure loss between the upper and lower punch can easily be estimated for different coefficients of wall friction. 

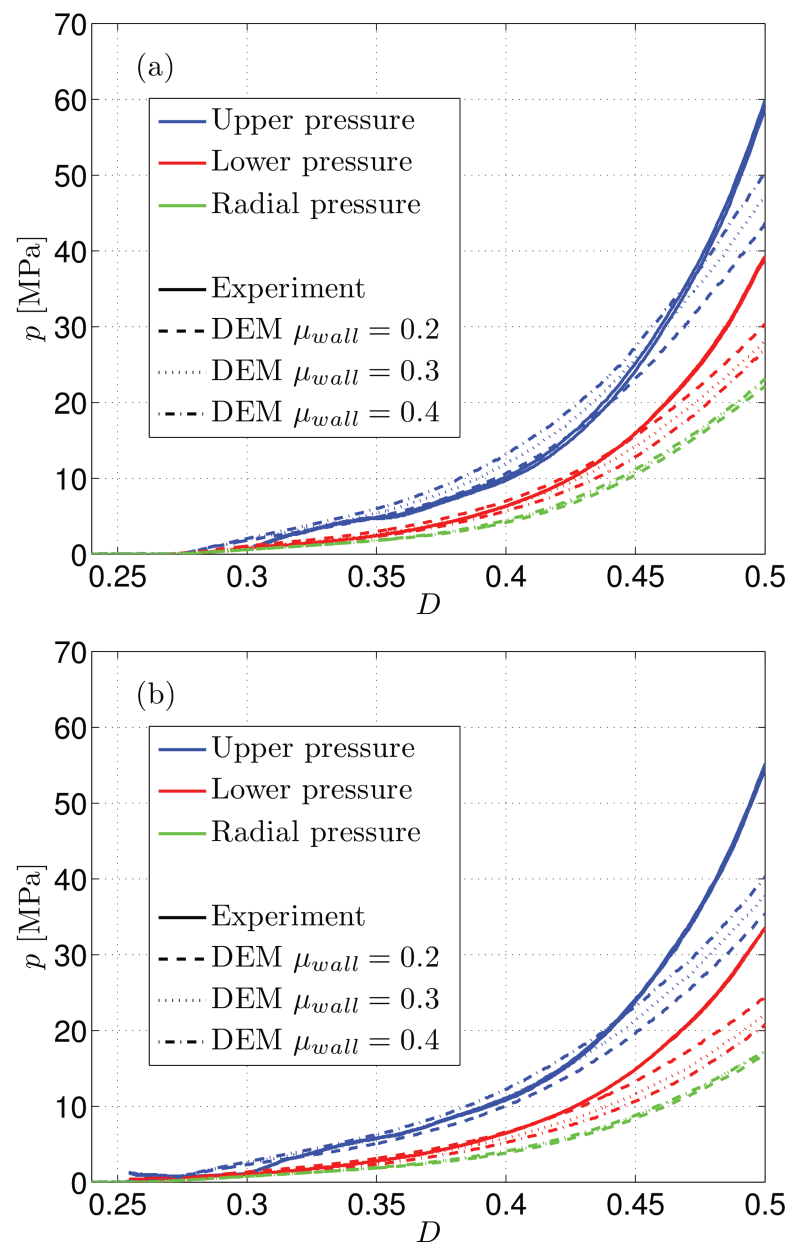

Figure 11: (a) Comparison between the DEM simulations and the results from the compaction experiments for (a) Powder A and (b) Powder B.

\section{Concluding remarks}

The compaction behavior of cemented carbide granules has been investigated using the discrete element method together with experiments performed on single powder granules in order to determine the constitutive behavior of the granules. This in contrast to previous studies of powder compaction where the constitutive description of the powder particles is assumed to be known, cf. eg. [11, 13, 31], and thus limits the applicability of such an analysis.

The mechanical behavior at low strains was investigated using a compression test on single granules showing a behavior that could be modeled by ideal plasticity with negligible elastic deformation. This is not in agreement with the compaction tests where a strong strain hardening behavior is seen. Hence, it is judged that the properties given from the granule compression tests are mostly influenced of the polymeric binder. Furthermore, the self-similarity solution of contacting plastic spheres is only an accurate solution for very 
small indentation depths but is useful for determining the plastic properties of the granules at low strains. For larger deformations, the finite element method needs to be relied upon.

The mechanical behavior at high strains was investigated using nanoindentation testing showing a strong strain hardening behavior as expected. This strong hardening can be explained by the fact that the small tungsten carbide and cobalt particles are coming into contact with each other, giving a close to discontinuous stress-strain response.

The proposed material model contains two parameters that could not be determined from the micromechanical experiments, the power law hardening exponent $m$ and the value of plastic strain when the hardening occurs, $\varepsilon_{H}$. The exact value of $m$ does not seem to be that important as long as it is sufficiently high to give a very strong hardening. The value of $\varepsilon_{H}$ is much more important but possibly $\varepsilon_{H}$ could be correlated by the density of the granules but this issue is left for further studies. Nevertheless, the micromechanical experiments gives a lot of information of the material behavior and reduces the number of unknown parameters significantly.

The discrete element method works well to predict the compaction behavior up to relative material densities of 0.45 in this case corresponding to a packing density of $90 \%$. At higher densities, the real response is stiffer then the results from the simulations. This is due to the fact that independent contact sites are assumed in DEM which fails at higher densities when contacts starts to interact. At these high densities, a continuum approach is more suitable.

\section{Acknowledgement}

The work was performed within the VINN Excellence Center Hero-m, financed by VINNOVA, the Swedish Governmental Agency for Innovation Systems, Swedish industry, and KTH Royal Institute of Technology. The authors would like to thank Stefan G Larsson, Per Lindskog, Carl-Johan Maderud, Daniel Petrini and Anders Stenberg at Sandvik AB for excellent experimental assistance and for providing the material used in the experiments. The authors also would like to thank Valter Ström at KTH MSE for performing the nanoindentation testing.

\section{References}

[1] D. Wilkinsson, M. F. Ashby, Pressure Sintering by Power Law Creep, Acta Metallurgica 23 (11) (1975) 1277-1285.

[2] N. A. Fleck, L. T. Kuhn, R. M. McMeeking, Yielding of Metal Powder Bonded by Isolated Contacts, Journal of the Mechanics and Physics of Solids 43 (9) (1992) 1139-1162.

[3] N. A. Fleck, On the Cold Compaction of Powders, Journal of the Mechanics and Physics of Solids 43 (9) (1995) $1409-1431$.

[4] S. Biwa, B. Storåkers, Analysis of Fully Plastic Brinell Indentation, Journal of the Mechanics and Physics of Solids 43 (8) (1995) 1303-1333.

[5] B. Storåkers, S. Biwa, P.-L. Larsson, Similarity Analysis of Inelastic Contact, International Journal of Solids and Structures 34 (24) (1997) 3061-3083.

[6] B. Storåkers, Local Contact Behaviour of Visco-plastic Particles, in: Proceedings of IUTAM Symposium Mechanics of Granular Flow and Particle Compaction, Kluwer Academic Publishers, Amsterdam, 1997, pp. 173-184.

[7] P.-L. Larsson, S. Biwa, B. Storåkers, Analysis of Cold and Hot Isostatic Compaction, Acta Materialia 44 (9) (1996) $3655-3666$. 
[8] B. Storåkers, N. A. Fleck, R. M. McMeeking, The Visco-plastic Compaction of Composite Powders, Journal of the Mechanics and Physics of Solids 47 (4) (1999) 785-815.

[9] S. D. Mesarovic, K. L. Johnson, Adhesive Contact of Elastic-Plastic Spheres, Journal of the Mechanics and Physics of Solids 48 (10) (2000) 2009-2033.

[10] O. Skrinjar, P.-L. Larsson, B. Storåkers, Local Contact Compliance Relations at Compaction of Composite Powders, Journal of Applied Mechanics 74 (1) (2007) 164-168.

[11] E. Olsson, P. L. Larsson, On Force-Displacement at Contact Between Elastic-Plastic Adhesive Bodies, submitted for publication.

[12] P. R. Heylinger, R. M. McMeeking, Cold Plastic Compaction of Powders by a Network Model, Journal of the Mechanics and Physics of Solids 49 (9) (2001) 2031-2054.

[13] C. L. Martin, D. Bouvard, S. Shima, Study of Particle Rearrangement During Powder Compaction by the Discrete Element Method, Journal of the Mechanics and Physics of Solids 51 (4) (2003) 667-693.

[14] C. L. Martin, D. Bouvard, Study of the Cold Compaction of Composite Powders by the Discrete Element Method, Acta Materialia 51 (2) (2003) 373-386.

[15] O. Skrinjar, P.-L. Larsson, On Discrete Element Modelling of Compaction of Powders with Size Ratio, Computional Materials Science 31 (1-2) (2004) 131-146.

[16] O. Skrinjar, P.-L. Larsson, Cold Compaction of Composite Powders with Size Ratio, Acta Materialia 57 (7) (2004) 18711884 .

[17] P. Pizette, C. L. Martin, G. Delette, P. Sornay, F. Sans, Compaction of Aggregated Ceramic Powders: from Contact Law to Fracture and Yield Surfaces, Powder Technology 198 (2) (2010) 240-250.

[18] http://www.etewe.de/etewe-granulatfestigkeit/pruefsysteme.html.

[19] ABAQUS, Abaqus 6.9, Dassault Systèmes Simulia Corp., Providence, RI, USA (2009).

[20] S. Carlsson, P.-L. Larsson, S. Biwa, On Frictional Effects at Inelastic Contact Between Spherical Bodies, International Journal of Mechanical Siences 42 (2000) 107-128.

[21] S. D. Mesarovic, N. A. Fleck, Spherical Indentation of Elastic-Plastic Solids, Proceedings of the Royal Society A 455 (1987) (1999) 2707-2728.

[22] S. D. Mesarovic, N. A. Fleck, Frictionless Indentation of Dissimilar Elstic-plastic Spheres, International Journal of Solids and Structures 37 (46-47) (2000) 7071-7091.

[23] D. C. Andersson, P. L. Larsson, A. Cadario, P. Lindskog, On the Influence from Punch Geometry on the Stress Distribution at Powder Compaction, Powder Technology 202 (2010) 78-88.

[24] P. Lindskog, D. C. Andersson, P. L. Larsson, An Experimental Device for Material Characterization of Powder Materials, Journal of Testing and Evaluation 41 (3) (2012) . doi:10.1520/JTE20120107.

[25] P.-L. Larsson, A. E. Giannakopoulos, E. Söderlund, D. J. Rowcliffe, R. Vestergaard, Analysis of Berkovic Indentation, International Journal of Solids and Structures 33 (2) (1996) 221-248.

[26] W. C. Oliver, G. M. Pharr, An Improved Technique for Determining Hardness and Elastic Modulus using Load and Displacement Sensing Experiements, Journal of Materials Research 7 (06) (1992) 1564-1583.

[27] T. Y. Tsui, W. C. Oliver, G. M. Pharr, Influences of Stress on the Measurement of Mechanical Properties using Nanoindentation: Part i. Experimental Studies in an Aluminum Alloy, Journal of Materials Research 11 (03) (1996) 752-759.

[28] P.-L. Larsson, Investigation of Sharp Contact ar Rigid-Plastic Conditions, International Journal of Mechanical Sciences 43 (4) (2001) 895-920.

[29] http://www.kzkptc.com/index.htm, accessed: 19/12/2012.

[30] P. A. Cundall, O. D. L. Strack, A Discrete Numerical Model for Granular Assemblies, Geotechnique 29 (1979) 49-62.

[31] E. Olsson, P.-L. Larsson, On the Effect of Particle Size Distribution in Cold Powder Compaction, Journal of Applied Mechanics 79 (5) (2012).

[32] C. Thornton, S. J. Antony, Quasi-Static Deformation of Particulate Media, Philosophical Transactions: Mathematical, Physical and Engineering Sciences 356 (1747) (1998) 2763-2782.

[33] P. Samuelson, B. Bolin, Experimental Studies of Frictional Behaviour of Hard Metal Powders Sliding on Cemented Carbide Walls, Scandinavian Journal of Metallurgy 12 (1983) 315-322.

[34] B. Wikman, N. Solimannezhad, R. Larsson, M. Oldenburg, H. Å. Häggblad, Wall Friction Coefficient Estimation by Modelling of a Powder Die Pressing Experiment, Powder Metallurgy 43 (2) (2000) 132-138. 